MATHEMATICS OF COMPUTATION

Volume 74, Number 249, Pages 341-362

S 0025-5718(04)01672-2

Article electronically published on May 25, 2004

\title{
REAL ORTHOGONAL POLYNOMIALS IN FREQUENCY ANALYSIS
}

\author{
C. F. BRACCIALI, XIN LI, AND A. SRI RANGA
}

\begin{abstract}
We study the use of para-orthogonal polynomials in solving the frequency analysis problem. Through a transformation of Delsarte and Genin, we present an approach for the frequency analysis by using the zeros and Christoffel numbers of polynomials orthogonal on the real line. This leads to a simple and fast algorithm for the estimation of frequencies. We also provide a new method, faster than the Levinson algorithm, for the determination of the reflection coefficients of the corresponding real Szegö polynomials from the given moments.
\end{abstract}

\section{INTRODUCTION}

Let $\nu$ be a positive measure on the unit circle. This means that the associated distribution function (still denoted by $\nu) \nu\left(e^{i \theta}\right):=\nu\left(\left\{e^{i t} \mid 0 \leq t<\theta\right\}\right.$ ), defined on $0 \leq \theta \leq 2 \pi$, is a real, bounded and nondecreasing function with infinitely many points of increase. Then the moments

$$
\mu_{m}^{(\nu)}=\int z^{m} d \nu(z)=\int_{0}^{2 \pi} e^{i m \theta} d \nu\left(e^{i \theta}\right), \quad m=0,1,2, \ldots,
$$

all exist. We consider the Szegö polynomials $\left\{S_{n}(\nu, z)\right\}$ associated with the measure $\nu$ defined by

$$
\int S_{n}(\nu, z) \overline{S_{m}(\nu, z)} d \nu(z)=0, \quad n \neq m .
$$

These polynomials were introduced by Szegő. See, for example, 19, 20, for interesting basic information on these polynomials.

The Szegö polynomials are known to satisfy the system of recurrence relations (here we write the polynomials in monic form)

$$
\begin{aligned}
S_{n+1}(\nu, z) & =z S_{n}(\nu, z)+a_{n+1}^{(\nu)} S_{n}^{*}(\nu, z), \\
\left(1-\left|a_{n+1}^{(\nu)}\right|^{2}\right) z S_{n}(\nu, z) & =S_{n+1}(\nu, z)-a_{n+1}^{(\nu)} S_{n+1}^{*}(\nu, z),
\end{aligned}
$$

Received by the editor March 8, 2003 and, in revised form, August 14, 2003.

2000 Mathematics Subject Classification. Primary 42C05, 94A11, 94A12.

Key words and phrases. Frequency analysis problem, frequency estimation, orthogonal polynomials, Szegő polynomials, para-orthogonal polynomials, quadrature.

This research was started while the second author was visiting the campus of UNESP at São José do Rio Preto, during September/October 2002, with a Fellowship from FAPESP. The first and the third authors' research is supported by grants from CNPq and FAPESP.

(C)2004 American Mathematical Society 
for $n \geq 0$. Here $S_{n}^{*}(\nu, z)=z^{n} \bar{S}_{n}(\nu, 1 / z)$ are the reciprocal polynomials. The numbers $a_{n}^{(\nu)}=S_{n}(\nu, 0), n \geq 1$, are known as the reflection coefficients of the Szego" polynomials.

The reflection coefficients have the property $\left|a_{n}^{(\nu)}\right|<1$ for $n \geq 1$. Furthermore, the zeros $\left\{S_{n}(\nu, z)\right\}$ are all inside the open unit disk.

The Wiener-Levinson method [21, 15] for the frequency analysis problem is based on the behaviour of the zeros of the Szegö polynomials associated with the positive absolutely continuous measure $\frac{1}{2 \pi}\left|\sum_{m=0}^{N-1} x(m) e^{-i m \theta}\right|^{2} d \theta$. Here $x(m)$ is a trigonometric signal, which we write

$$
x(m)=\gamma e^{i m \pi}+\sum_{j=1}^{I}\left(\gamma_{j} e^{i m \omega_{j}}+\gamma_{n_{0}+1-j} e^{i m \omega_{n_{0}+1-j}}\right),
$$

where $n_{0}=2 I+1$ if $\gamma>0$ and $n_{0}=2 I$ if $\gamma=0$.

When $\gamma>0$, set $\gamma_{I+1}=\gamma$ and $\omega_{I+1}=\pi$. One can assume

$$
0<\omega_{1}<\omega_{2}<\cdots<\omega_{n_{0}-1}<\omega_{n_{0}}<2 \pi
$$

and

$$
\overline{\gamma_{n_{0}+1-j}}=\gamma_{j} \neq 0, \quad 2 \pi-\omega_{n_{0}+1-j}=\omega_{j} \neq 0, \quad \text { for } \quad j=1,2, \ldots, I .
$$

The constants $\gamma_{j}$ are known as the (complex) amplitudes of the signal, $\omega_{j}$ are known as the frequencies and $m$ represents discrete time. The frequency analysis problem is to determine the unknowns $n_{0}, \gamma_{j}$ and $\omega_{j}$ from observed values of $x(m), m=$ $0,1,2, \ldots$.

Let $\lambda_{k}=\left|\gamma_{k}\right|^{2}, k=1,2, \ldots, n_{0}$, and let $\psi$ be the discrete measure whose distribution function is given by

$$
\psi\left(e^{i \theta}\right)=\sum_{k=1}^{n_{0}} \lambda_{k} H\left(\theta-\omega_{k}\right), \quad 0 \leq \theta<2 \pi .
$$

Here, and from now on, $H(x)$ is the Heaviside function defined by $H(x)=1$ for $x \geq 0$ and $H(x)=0$ for $x<0$.

The measure $\psi$ has precisely $n_{0}$ points of increase and the associated Szego" polynomials $S_{k}(\psi, z)$ exist uniquely only for $k=1,2, \ldots, n_{0}$.

Let the absolutely continuous measure $\psi_{N}$ be given by

$$
\frac{d \psi_{N}\left(e^{i \theta}\right)}{d \theta}=\frac{1}{2 \pi N}\left|\sum_{m=0}^{N-1} x(m) e^{-i m \theta}\right|^{2}, \quad 0 \leq \theta<2 \pi
$$

Then the following results are known.

Theorem A. We have

(1) The measure $\psi_{N}$ converges in the weak-star topology to the discrete measure $\psi$. This means

$$
\lim _{N \rightarrow \infty} \int_{0}^{2 \pi} f\left(e^{i \theta}\right) d \psi_{N}\left(e^{i \theta}\right)=\int_{0}^{2 \pi} f\left(e^{i \theta}\right) d \psi\left(e^{i \theta}\right),
$$

for all $f$ continuous on the unit circle. 
(2) For each fixed $n, 1 \leq n \leq n_{0}$,

$$
\lim _{N \rightarrow \infty} S_{n}\left(\psi_{N}, z\right)=S_{n}(\psi, z), \quad z \in \mathbb{C},
$$

where $S_{n}(\psi, z)$ is the monic $n$-th degree Szegö polynomial associated with the discrete measure $\psi$. In particular,

$$
\lim _{N \rightarrow \infty} S_{n_{0}}\left(\psi_{N}, z\right)=S_{n_{0}}(\psi, z)=\prod_{m=1}^{n_{0}}\left(z-\zeta_{m}\right), \quad z \in \mathbb{C},
$$

where $\zeta_{m}=e^{i \omega_{m}}$.

(3) For each $n \neq n_{0}$, there is an $L_{n} \in(0,1)$, depending on $n$ only, such that $\left|S_{n}\left(\psi_{N}, 0\right)\right|=\left|a_{n}^{(N)}\right| \leq L_{n}<1, \quad N=1,2, \ldots$

(4) For each fixed $n>n_{0}$, the $n_{0}$ zeros of $S_{n}\left(\psi_{N}, z\right)$ of largest modulus approach the points $\zeta_{m}, m=1,2, \ldots, n_{0}$. In addition, there exists a number $K_{n}<1$, depending only on $n$, such that the remaining $n-n_{0}$ of $S_{n}\left(\psi_{N}, z\right)$ are inside the disk $|z| \leq K_{n}$.

For the first two results see [9]. For the last two see [11, 16, 17]. For a recent survey of the applications of Szegö polynomials in frequency analysis we refer to [13.

Hence the problem of finding the frequencies $\omega_{j}$ can be treated by constructing the polynomials $\left\{S_{n}\left(\psi_{N}, z\right)\right\}_{n=0}^{\infty}$ with growing $N$ and then by observing the behavior of their zeros.

When $N$ is fixed, for the construction of $\left\{S_{n}\left(\psi_{N}, z\right)\right\}_{n=0}^{\infty}$ one might use the Levinson algorithm, which can be given as follows.

Let $S_{k}\left(\psi_{N}, z\right)=\sum_{j=0}^{k} s_{j, k} z^{j}$ and $a_{k+1}^{(N)}=S_{k+1}\left(\psi_{N}, 0\right)$, for $k=0,1, \ldots$

Algorithm I (The Levinson algorithm).

\section{Set}

$$
\begin{aligned}
& \rho_{0}=\int_{0}^{2 \pi} d \psi_{N}\left(e^{i \theta}\right)=\mu_{0}^{(N)}, \quad s_{0,0}=1 ; \\
& a_{1}^{(N)}=-\frac{1}{\rho_{0}} \int_{0}^{2 \pi} e^{-i \theta} d \psi_{N}\left(e^{i \theta}\right)=-\frac{\mu_{1}^{(N)}}{\rho_{0}}, \quad s_{0,1}=a_{1}^{(N)}, \quad s_{1,1}=1 .
\end{aligned}
$$

For $k=1,2,3, \ldots, n-1$, do

$$
\begin{aligned}
& \rho_{k}=\left(1-\left|a_{k}^{(N)}\right|^{2}\right) \rho_{k-1}, \\
& a_{k+1}^{(N)}=-\frac{1}{\rho_{k}} \int_{0}^{2 \pi} \frac{e^{i \theta} S_{k}\left(e^{i \theta}\right)}{d} d \psi_{N}\left(e^{i \theta}\right)=-\frac{1}{\rho_{k}} \sum_{j=0}^{k} s_{j, k} \mu_{j+1}^{(N)}, \\
& s_{0, k+1}=a_{k+1}^{(N)}, s_{k+1, k+1}=1, s_{j, k+1}=s_{j-1, k}+a_{k+1}^{(N)} s_{k-j, k}, j=1,2, \ldots, k .
\end{aligned}
$$

The moments $\mu_{k}^{(N)}=\int_{0}^{2 \pi} e^{i k \theta} d \psi_{N}\left(e^{i \theta}\right)$ are generated by $\mu_{-k}^{(N)}=\mu_{k}^{(N)}$ and

$$
\mu_{k}^{(N)}=\frac{1}{N} \sum_{m=k}^{N-1} x(m) x(m-k) \quad \text { for } \quad k=0,1, \ldots
$$

The sums on the right-hand side are also called the autocorrelation coefficients of the signal.

Though we have found the polynomials $\left\{S_{n}\left(\psi_{N}, z\right)\right\}_{n=0}^{\infty}$, the evaluation of their zeros (complex and lying inside the open unit disk) still presents much difficulty. We cite the work of Ammar, Calvetti and Reichel [1], where they determine first 
the zeros of a neighboring polynomial which is the characteristic polynomial of a unitary upper Hessenberg matrix. The zeros of the Szegö polynomials are then determined by a continuation method.

Yet another possible way is to use, instead, the para-orthogonal polynomials, where finding zero is essentially an eigenvalue problem associated with a unitary matrix since all the zeros of the para-orthogonal polynomials are on the unit circle. The main difficulty now is how to distinguish between the interesting zeros (those approaching the frequency points) and the "uninteresting" ones. Jones, Njåstad and Waadeland 12 were the first to use the para-orthogonal polynomials in solving the frequency analysis problem. The recent work by Daruis, Njåstad and Van Assche [5] illustrated how the quadrature weights associated with the para-orthogonal polynomials can be used to find out frequency points. In this paper, we first further study the asymptotic behavior of the zeros of the para-orthogonal polynomials and the quadrature weights. Then we show how to simultaneously use two families of para-orthogonal polynomials to help us find the interesting zeros. In particular, we will show that special para-orthogonal polynomials can be chosen so that the whole problem becomes the evaluation of the zeros and Christoffel numbers associated with real orthogonal polynomials on $[-1,1]$.

\section{PARA-ORThogonal POlynomials}

In [10], Jones, Njåstad and Thron initiated a study of the polynomials

$$
S_{n}(\nu, \tau, z)=S_{n}(\nu, z)+\tau S_{n}^{*}(\nu, z), \quad n \geq 1
$$

where $|\tau|=1$, which they called para-orthogonal polynomials (associated with the measure $\nu$ and the parameter $\tau$ ). Clearly, for $n \geq 2$, these polynomials satisfy

$$
\int_{\mathcal{C}} S_{n}(\nu, \tau, z) \overline{z^{k}} d \nu(z)=\int_{0}^{2 \pi} S_{n}\left(\nu, \tau, e^{i \theta}\right) e^{-i k \theta} d \nu\left(e^{i \theta}\right)=0, \quad 1 \leq k \leq n-1 .
$$

The word "para" is used to indicate the deficiency in the above orthogonality property (unlike the corresponding Szegö polynomial, $S_{n}(\nu, \tau, z)$ lacks the property for $k=0)$. However, $S_{n}(\nu, \tau, z)$ has $n$ simple zeros $z_{1, n}(\nu, \tau), z_{2, n}(\nu, \tau), \ldots, z_{n, n}(\nu, \tau)$, all lying on the unit circle.

Now let us say that $f \in \Omega_{n-1}$ if $f(z) \in \operatorname{Span}\left\{z^{-n+1}, z^{-n+2}, \ldots, z^{n-2}, z^{n-1}\right\}$ for $|z|=1$. Then the quadrature rule

$$
\int_{0}^{2 \pi} f\left(e^{i \theta}\right) d \nu\left(e^{i \theta}\right)=\sum_{m=1}^{n} \lambda_{m, n}(\nu, \tau) f\left(z_{m, n}(\nu, \tau)\right)
$$

holds for $f \in \Omega_{n-1}$, if

$$
\lambda_{m, n}(\nu, \tau)=\int_{0}^{2 \pi} \frac{S_{n}\left(\nu, \tau, e^{i \theta}\right)}{\left(e^{i \theta}-z_{m, n}(\nu, \tau)\right) S_{n}^{\prime}\left(\nu, \tau, z_{m, n}(\nu, \tau)\right)} d \nu\left(e^{i \theta}\right), m=1,2, \ldots, n .
$$

For given $|\tau|=1$ and $n \geq 1$, we now consider the polynomials $Q_{m}(n, \nu, \tau, z)$ given by the recurrence relation

$Q_{m}(n, \nu, \tau, z)=z Q_{m-1}(n, \nu, \tau, z)+z \tau \overline{a_{n+1-m}^{(\nu)}} Q_{m-1}^{*}(n, \nu, \tau, z), \quad m=1,2, \ldots, n$,

with $Q_{0}(n, \nu, \tau, z)=Q_{0}^{*}(n, \nu, \tau, z)=1$. Here $a_{n}^{(\nu)}=S_{n}(\nu, 0)$.

The polynomial $Q_{m}(n, \nu, \tau, z)$ is monic and of degree $m$. From the recurrence relations of $Q_{m}(n, \nu, \tau, z)$ and $S_{m}(\nu, z)$, we obtain 
Theorem 2.1. The following statements hold.

(1) The $m$ zeros of $Q_{m}(n, \nu, \tau, z)$ are all inside the open unit disk $|z|<1$.

(2) For any $z$ on the closed unit disk $|z| \leq 1$,

$$
\begin{gathered}
\left|Q_{m}(n, \nu, \tau, z)\right| \leq|z| \prod_{j=1}^{m}\left(1+\left|a_{n+1-j}^{(\nu)}\right|\right), \\
0<\prod_{j=1}^{m}\left(1-\left|a_{n+1-j}^{(\nu)}\right|\right) \leq\left|Q_{m}^{*}(n, \nu, \tau, z)\right| \leq \prod_{j=1}^{m}\left(1+\left|a_{n+1-j}^{(\nu)}\right|\right),
\end{gathered}
$$

for $m=1,2, \ldots, n$.

(3) $\quad S_{n}(\nu, \tau, z)=Q_{m}(n, \nu, \tau, z) S_{n-m}(\nu, z)+\tau Q_{m}^{*}(n, \nu, \tau, z) S_{n-m}^{*}(\nu, z)$,

for $m=1,2, \ldots, n$.

In [5], Daruis, Njåstad and Van Assche realized that the size of the weights in the quadratures associated with a para-orthogonal polynomial can be used for studying the frequency analysis problem. Consider the polynomial

$$
S_{n}\left(\psi_{N}, \tau, z\right)=S_{n}\left(\psi_{N}, z\right)+\tau S_{n}^{*}\left(\psi_{N}, z\right),
$$

with $|\tau|=1$, which is a para-orthogonal polynomial associated with the measure $\psi_{N}$. For the zeros of $S_{n}\left(\psi_{N}, \tau, z\right)$ and the corresponding quadrature weights, we use the notation

$$
z_{m, n}(N, \tau)=e^{i \theta_{m, n}(N, \tau)} \quad \text { and } \quad \lambda_{m, n}(N, \tau), \quad m=1,2, \ldots, n .
$$

The following results were established in [5].

Theorem B ([5, Theorems 1.1 and 2.1]).

1) For any fixed $n \geq 1$,

$$
\lim _{N \rightarrow \infty} \sum_{m=1}^{n} \lambda_{m, n}(N, \tau)=\sum_{m=1}^{n_{0}} \lambda_{m}(\psi),
$$

where $\lambda_{m}(\psi)=\lambda_{m}$ are the weights appearing in (3).

2) Let $n \geq n_{0}$ be fixed. Let $\Lambda$ be an arbitrary subsequence of the sequence of natural numbers, and let $\tau$ be an arbitrary point such that $|\tau|=1$. Then there exists a subsequence $\Lambda_{1}$ of $\Lambda$ and a polynomial $W_{n-n_{0}}(\tau, z)$ of degree $n-n_{0}$ such that

$$
\lim _{\substack{N \rightarrow \infty \\ N \in \Lambda_{1}}} S_{n}\left(\psi_{N}, \tau, z\right)=W_{n-n_{0}}(\tau, z) S_{n_{0}}(\psi, z) .
$$

Now we denote the distinct zeros of $S_{n_{0}}(\psi, z)$ by $\zeta_{1}, \zeta_{2}, \ldots, \zeta_{n_{0}}$ and the zeros of $W_{n-n_{0}}(\tau, z)$ by $\zeta_{n_{0}+1}(\tau), \zeta_{n_{0}+2}(\tau), \ldots, \zeta_{n}(\tau)$. We can assume, without loss of generality, that

$$
\begin{aligned}
& \lim _{\substack{N \rightarrow \infty \\
N \in \Lambda_{1}}} z_{m, n}(N, \tau)=\zeta_{m}, \quad \text { for } m=1,2, \ldots, n_{0}, \\
& \lim _{\substack{N \rightarrow \infty \\
N \in \Lambda_{1}}} z_{m, n}(N, \tau)=\zeta_{m}(\tau), \quad \text { for } m=n_{0}+1, \ldots, n .
\end{aligned}
$$

Any of the zeros $\zeta_{n_{0}+1}(\tau), \zeta_{n_{0}+2}(\tau), \ldots, \zeta_{n}(\tau)$ of $W_{n-n_{0}}(\tau, z)$ may or may not coincide with the frequency points (zeros $\zeta_{1}, \zeta_{2}, \ldots, \zeta_{n_{0}}$ of $S_{n_{0}}(\psi, z)$ ).

The following results are also given in [5]. 
Theorem C ([5. Theorems 3.1, 4.1, and 5.2, Corollary 5.3]). With the notation introduced above, we have

(1) If the subsequence $\left\{z_{m, n}(N, \tau): N \in \Lambda_{1}\right\}$ converges to a value different from a frequency point, then

$$
\lim _{\substack{N \rightarrow \infty \\ N \in \Lambda_{1}}} \lambda_{m, n}(N, \tau)=0
$$

(2) Suppose that $\zeta_{n_{0}+m}(\tau)=\zeta_{m}$ for $m=1, \ldots, p$ and that $\zeta_{1}, \ldots, \zeta_{n_{0}}$, $\zeta_{n_{0}+p+1}(\tau), \ldots, \zeta_{n}(\tau)$ are distinct points. Then

$$
\begin{array}{cl}
\lim _{\substack{N \rightarrow \infty \\
N \in \Lambda_{1}}}\left[\lambda_{m, n}(N, \tau)+\lambda_{n_{0}+m, n}(N, \tau)\right]=\lambda_{m}(\psi), & \text { for } m=1, \ldots, p, \\
\lim _{\substack{N \rightarrow \infty \\
N \in \Lambda_{1}}} \lambda_{m, n}(N, \tau)=\lambda_{m}(\psi), & \text { for } m=p+1, \ldots, n_{0}, \\
\lim _{\substack{N \rightarrow \infty \\
N \in \Lambda_{1}}} \lambda_{m, n}(N, \tau)=0, & \text { for } m=n_{0}+p+1, \ldots, n,
\end{array}
$$

(3) In particular, if the zeros of $W_{n-n_{0}}(\tau, z) S_{n_{0}}(\psi, z)$ are simple, then

$$
\begin{array}{ll}
\lim _{\substack{N \rightarrow \infty \\
N \in \Lambda_{1}}} \lambda_{m, n}(N, \tau)=\lambda_{m}(\psi), \quad \text { for } m=1,2, \ldots, n_{0}, \\
\lim _{\substack{N \rightarrow \infty \\
N \in \Lambda_{1}}} \lambda_{m, n}(N, \tau)=0, \quad \text { for } m=n_{0}+1, \ldots, n .
\end{array}
$$

We can now give some results that improve and complement Theorems A-C above. First, we have an analogue of Theorem A(3)-(4) for the para-orthogonal polynomials.

Theorem 2.2. Let $n>n_{0}$ be fixed. Then, for any $\epsilon>0$, there exists an $N(\epsilon)$ such that, for all $N \geq N(\epsilon)$, each one of the arcs $\left\{z:|z|=1\right.$ and $\left.\left|z-\zeta_{m}\right|<\epsilon\right\}$, $m=1,2, \ldots, n_{0}$, contains at least one zero of $S_{n}\left(\psi_{N}, \tau, z\right)$.

Proof. We have from Theorem 2.1 by substituting $a_{n}^{(\nu)}$ by $a_{n}^{(N)}$

$$
S_{n}\left(\psi_{N}, \tau, z\right)=Q_{n-n_{0}}\left(n, \psi_{N}, \tau, z\right) S_{n_{0}}\left(\psi_{N}, z\right)+\tau Q_{n-n_{0}}^{*}\left(n, \psi_{N}, \tau, z\right) S_{n_{0}}^{*}\left(\psi_{N}, z\right) .
$$

Hence, if we consider the integral $\int\left|S_{n}\left(\psi_{N}, \tau, z\right)\right| d \psi(z)$, we obtain

$$
\begin{aligned}
& \int\left|S_{n}\left(\psi_{N}, \tau, z\right)\right| d \psi(z) \\
& \quad=\sum_{m=1}^{n_{0}} \lambda_{m}\left|S_{n_{0}}\left(\psi_{N}, \zeta_{m}\right)\right|\left|Q_{n-n_{0}}\left(n, \psi_{N}, \tau, \zeta_{m}\right)+\tau_{m} Q_{n-n_{0}}^{*}\left(n, \psi_{N}, \tau, \zeta_{m}\right)\right|,
\end{aligned}
$$

where $\tau_{m}=\tau S_{n_{0}}^{*}\left(\psi_{N}, \zeta_{m}\right) / S_{n_{0}}\left(\psi_{N}, \zeta_{m}\right)$ for $m=1,2, \ldots, n_{0}$. Note that $\left|\tau_{m}\right|=1$ for $m=1,2, \ldots, n_{0}$. Thus from the inequalities in Theorem 2.1]

$$
\int\left|S_{n}\left(\psi_{N}, \tau, z\right)\right| d \psi(z) \leq 2\left\{\prod_{j=1}^{n-n_{0}}\left(1+\left|a_{n+1-j}^{(N)}\right|\right)\right\} \sum_{m=1}^{n_{0}} \lambda_{m}\left|S_{n_{0}}\left(\psi_{N}, \zeta_{m}\right)\right| .
$$


Since $\lim _{N \rightarrow \infty} S_{n_{0}}\left(\psi_{N}, z\right)=S_{n_{0}}(\psi, z)$ and $\left|a_{n+1-j}^{(N)}\right|<1$ for $j=1,2, \ldots, n-n_{0}$, we obtain

$$
\lim _{N \rightarrow \infty} \sum_{m=1}^{n_{0}} \lambda_{m}\left|S_{n}\left(\psi_{N}, \tau, \zeta_{m}\right)\right|=\lim _{N \rightarrow \infty} \int\left|S_{n}\left(\psi_{N}, \tau, z\right)\right| d \psi(z)=0,
$$

which implies the theorem.

The above theorem guarantees that for each $N$ we can pick out (distinct) zeros $z_{m, n}(N, \tau), m=1,2, \ldots, n_{0}$, of $S_{n}\left(\psi_{N}, \tau, z\right)$ such that $\lim _{N \rightarrow \infty} z_{m, n}(N, \tau)=\zeta_{m}$.

Before we state our next result, let us make a simple but useful observation. For any $f(z) \in \Omega_{n-1}$, using the quadrature (4) with $\nu=\psi_{N}$, we get

$$
\sum_{m=1}^{n} \lambda_{m, n}(N, \tau) f\left(z_{m, n}(N, \tau)\right)=\int f(z) d \psi_{N}(z)
$$

which converges to $\int f(z) d \psi(z)$ since $\psi_{N}(z) \stackrel{w *}{\longrightarrow} \psi(z)$ as $N \rightarrow \infty$. Thus, for every $f(z) \in \Omega_{n-1}$,

$$
\lim _{N \rightarrow \infty} \sum_{m=1}^{n} \lambda_{m, n}(N, \tau) f\left(z_{m, n}(N, \tau)\right)=\int f(z) d \psi(z)
$$

We now show that (5) is not only true when $f \in \Omega_{n-1}$, but it is also valid for all continuous functions on the unit circle. Define a sequence of discrete measures $\psi_{N}(n, \tau, \cdot), N=1,2, \ldots$, obtained from the quadratures for the $\psi_{N}(z)$ :

$$
\psi_{N}\left(n, \tau, e^{i \theta}\right)=\sum_{m=1}^{n} \lambda_{m, n}(N, \tau) H\left(\theta-\theta_{m, n}(N, \tau)\right),
$$

where $z_{m, n}(N, \tau)=e^{i \theta_{m, n}(N, \tau)}$.

Theorem 2.3. Let $n>n_{0}$. Then, we have $\psi_{N}(n, \tau, z) \stackrel{w *}{\longrightarrow} \psi(z)$ as $N \rightarrow \infty$.

Proof. Note that $\left\{\psi_{N}(n, \tau, z)\right\}_{N=1}^{\infty}$ is weak-star compact. So, every subsequence of it contains a convergent sub-subsequence in the weak-star topology. Thus, to prove the theorem, it suffices to show that every convergent subsequence will have the same weak-star limit.

Assume $\psi_{N_{k}}(n, \tau, z) \stackrel{w *}{\longrightarrow} \tilde{\psi}(z)$ as $k \rightarrow \infty$ for some measure $\tilde{\psi}(z)$ on the unit circle. We show that $\tilde{\psi}(z)=\psi(z)$ in the following two steps.

Step 1. $\operatorname{supp}(\tilde{\psi}) \subseteq\left\{\zeta_{1}, \zeta_{2}, \ldots, \zeta_{n_{0}}\right\}$.

We show that $\tilde{\psi}(\Gamma)=0$ if $\Gamma$ is a closed arc that does not contain any $\zeta_{j}$ for $j=1,2, \ldots, n_{0}$. Let $\varepsilon>0$. There exists a continuous function $f_{\varepsilon}(z)$ on the unit circle such that $f_{\varepsilon}(z)=1$ if $z \in \Gamma,\left|f_{\varepsilon}(z)\right| \leq 1$ if $0<d(z, \Gamma)<\varepsilon$ and $f_{\varepsilon}(z)=0$ if $d(z, \Gamma) \geq \varepsilon$. Here $d(z, \Gamma)$ is the smallest distance of $z$ from $\Gamma$. Then,

$$
\int f_{\varepsilon}(z) d \tilde{\psi}(z)=\lim _{k \rightarrow \infty} \int f_{\varepsilon}(z) d \psi_{N_{k}}(n, \tau, z) .
$$


Note that when $\varepsilon$ is small enough, $d\left(\zeta_{j}, \Gamma\right) \geq 2 \varepsilon$ for $j=1,2, \ldots, n_{0}$, and so

$$
\begin{aligned}
\int f_{\varepsilon}(z) & d \psi_{N_{k}}(n, \tau, z)=\sum_{m=1}^{n} \lambda_{m, n}\left(N_{k}, \tau\right) f_{\varepsilon}\left(z_{m, n}\left(N_{k}, \tau\right)\right) \\
\leq & \sum_{d\left(z_{m, n}\left(N_{k}, \tau\right), \Gamma\right)<\varepsilon} \lambda_{m, n}\left(N_{k}, \tau\right) \frac{\prod_{j=1}^{n_{0}}\left|z_{m, n}\left(N_{k}, \tau\right)-\zeta_{j}\right|^{2}}{\prod_{j=1}^{n_{0}}\left|z_{m, n}\left(N_{k}, \tau\right)-\zeta_{j}\right|^{2}} \\
\leq & \frac{1}{\varepsilon^{2 n_{0}}} \sum_{d\left(z_{m, n}\left(N_{k}, \tau\right), \Gamma\right)<\varepsilon} \lambda_{m, n}\left(N_{k}, \tau\right) \prod_{j=1}^{n_{0}}\left|z_{m, n}\left(N_{k}, \tau\right)-\zeta_{j}\right|^{2} \\
\leq & \frac{1}{\varepsilon^{2 n_{0}}} \sum_{m=1}^{n} \lambda_{m, n}\left(N_{k}, \tau\right) \prod_{j=1}^{n_{0}}\left|z_{m, n}\left(N_{k}, \tau\right)-\zeta_{j}\right|^{2}
\end{aligned}
$$

which, by (5), is equal to $\varepsilon^{-2 n_{0}} \int\left|\prod_{j=1}^{n_{0}}\left(z-\zeta_{j}\right)\right|^{2} d \psi_{N_{k}}(z) \rightarrow 0$ as $k \rightarrow \infty$. It follows from this and (6) that

$$
\int f_{\varepsilon}(z) d \tilde{\psi}(z)=0
$$

for all small $\varepsilon>0$, which implies $\mu(\Gamma)=0$. This finishes the proof of Step 1 .

Step 2. $\tilde{\psi}\left(\left\{\zeta_{j}\right\}\right)=\lambda_{j}$ for $j=1,2, \ldots, n_{0}$.

Let $f_{p}(z):=S_{n_{0}}(\psi, z) /\left[\left(z-\zeta_{p}\right) S_{n_{0}}^{\prime}\left(\psi, \zeta_{p}\right)\right]$ for $p=1,2, \ldots, n_{0}$. Then

$$
\tilde{\psi}\left(\left\{\zeta_{p}\right\}\right)=\int\left|f_{p}(z)\right|^{2} d \tilde{\psi}(z)=\lim _{k \rightarrow \infty} \int\left|f_{p}(z)\right|^{2} d \psi_{N_{k}}(n, \tau, z),
$$

which, by (5), equals

$$
\lim _{k \rightarrow \infty} \int\left|f_{p}(z)\right|^{2} d \psi_{N_{k}}(z)=\int\left|f_{p}(z)\right|^{2} d \psi(z)=\sum_{j=1}^{n_{0}} \lambda_{j}\left|f_{p}\left(\zeta_{j}\right)\right|^{2}=\lambda_{p} .
$$

This finishes Step 2.

From Theorem 2.3, we can derive statements like those in Theorems B and C. In fact, we can make the following stronger statement.

Corollary 2.4. Let $\epsilon>0$ be such that the intervals $Y_{j}(\epsilon)=\left(\omega_{j}-\epsilon, \omega_{j}+\epsilon\right), j=$ $1,2, \ldots, n_{0}$, satisfy

$$
Y_{j}(\epsilon) \subset(0,2 \pi) \quad \text { and } \quad \omega_{k} \notin Y_{j}(\epsilon) \text { if } k \neq j .
$$

Set $\hat{Y}(\epsilon)=[0,2 \pi] \backslash \bigcup_{j=1}^{n_{0}} Y_{j}(\epsilon)$. Then,

$$
\begin{aligned}
& \lim _{N \rightarrow \infty} \sum_{\theta_{k, n}(N, \tau) \in Y_{j}(\epsilon)} \lambda_{k, n}(N, \tau)=\lambda_{j}, \quad j=1,2, \ldots, n_{0}, \\
& \lim _{N \rightarrow \infty} \sum_{\theta_{k, n}(N, \tau) \in \hat{Y}(\epsilon)} \lambda_{k, n}(N, \tau)=0 .
\end{aligned}
$$

Again, let $z_{m, n}(N, \tau), m=1,2, \ldots, n_{0}$, be the zeros of $S_{n}\left(\psi_{N}, \tau, z\right)$ that satisfy $\lim _{N \rightarrow \infty} z_{m, n}(N, \tau)=\zeta_{m}$ for $m=1,2, \ldots, n_{0}$, as guaranteed by Theorem 2.2 Define $V_{n_{0}}(N, \tau, z)=\prod_{m=1}^{n_{0}}\left[z-z_{m, n}(N, \tau)\right]$ and write

$$
S_{n}\left(\psi_{N}, \tau, z\right)=V_{n_{0}}(N, \tau, z) W_{n-n_{0}}(N, \tau, z) .
$$

Clearly, $\lim _{N \rightarrow \infty} V_{n_{0}}(N, \tau, z)=S_{n_{0}}(\psi, z)$. 
As mentioned earlier, the difficulty in using the para-orthogonal polynomials in the frequency analysis problem lies in how to distinguish interesting zeros from the uninteresting ones. In this aspect, Theorems B and $\mathrm{C}$ and the above corollary indicate how the weights can help. Our next result suggests that using two sequences of para-orthogonal polynomials is another feasible approach.

Theorem 2.5. Let $n>n_{0}$ be fixed. Let $\Lambda$ be an arbitrary subsequence of the sequence of natural numbers.

(1) Then there exists a subsequence $\Lambda_{1}$ of $\Lambda$ such that

$$
\lim _{\substack{N \rightarrow \infty \\ N \in \Lambda_{1}}} W_{n-n_{0}}\left(N, \tau_{1}, z\right)=W_{n-n_{0}}\left(\tau_{1}, z\right) .
$$

(2) If $\tau_{1} \neq \tau_{2}$, then there exists a subsequence $\Lambda_{2}$ of $\Lambda_{1}$ such that

$$
\begin{aligned}
& \lim _{\substack{N \rightarrow \infty \\
N \in \Lambda_{2}}} W_{n-n_{0}}\left(N, \tau_{1}, z\right)=W_{n-n_{0}}\left(\tau_{1}, z\right) \\
& \lim _{\substack{n \rightarrow \Lambda_{2} \\
N \in \Lambda_{2}}} W_{n-n_{0}}\left(N, \tau_{2}, z\right)=W_{n-n_{0}}\left(\tau_{2}, z\right) .
\end{aligned}
$$

Moreover, $W_{n-n_{0}}\left(\tau_{1}, z\right)$ and $W_{n-n_{0}}\left(\tau_{2}, z\right)$ cannot have common zeros.

Proof. The existence of $W_{n-n_{0}}\left(\tau_{1}, z\right)$ and $W_{n-n_{0}}\left(\tau_{2}, z\right)$ follows from the behavior of infinite sequences of bounded functions and adds nothing to what has already been shown in [5]; see Theorem B(2) above.

To prove the last statement, suppose that $W_{n-n_{0}}\left(\tau_{1}, z\right)$ and $W_{n-n_{0}}\left(\tau_{2}, z\right)$ share a common zero $\zeta$, which has to be on the unit circle. The point $\zeta$ can be equal or not equal to any of the frequency points. We write

$$
W_{n-n_{0}}\left(\tau_{1}, z\right)=(z-\zeta) \tilde{W}_{n-n_{0}-1}\left(\tau_{1}, z\right)
$$

and

$$
W_{n-n_{0}}\left(\tau_{2}, z\right)=(z-\zeta) \tilde{W}_{n-n_{0}-1}\left(\tau_{2}, z\right) .
$$

Hence from above and from (7), we obtain

$$
\begin{aligned}
& \lim _{\substack{N \rightarrow \infty \\
N \in \Lambda_{2}}}\left(\tau_{1}-\tau_{2}\right) S_{n}\left(\psi_{N}, z\right) \\
= & \lim _{\substack{N \rightarrow \infty \\
N \in \Lambda_{2}}}\left[\tau_{1} S_{n}\left(\psi_{N}, \tau_{2}, z\right)-\tau_{2} S_{n}\left(\psi_{N}, \tau_{1}, z\right)\right] \\
= & (z-\zeta) S_{n_{0}}(\psi, z)\left[\tau_{1} \tilde{W}_{n-n_{0}-1}\left(\tau_{2}, z\right)-\tau_{2} \tilde{W}_{n-n_{0}-1}\left(\tau_{1}, z\right)\right] .
\end{aligned}
$$

When $\tau_{1} \neq \tau_{2}$, this is a contradiction to the fact that $n-n_{0}$ zeros of $S_{n}\left(\psi_{N}, z\right)$ are inside the disk $|z| \leq K_{n}$ as pointed out in Section 1, Theorem A(4).

From this result, we see that between common convergent subsequences of

$$
\left\{S_{n}\left(\psi_{N_{k}}, 1, z\right)\right\}_{k=1}^{\infty} \text { and }\left\{S_{n}\left(\psi_{N_{k}},-1, z\right)\right\}_{k=1}^{\infty}
$$

the only common factor of their limits is $S_{n_{0}}(\psi, z)$. The above theorem can be used to find the interesting zeros by observing the asymptotic behavior of two distinct sequences of para-orthogonal polynomials (at least in theory). 


\section{REAL PARA-ORTHOGONAL POLYNOMIALS}

Let us assume in this section that the reflection coefficients are real, i.e., $-1<$ $a_{n}<1$. Note that the reflection coefficients are real if and only if the measure $d \nu(z)$ satisfies the symmetry $d \nu(1 / z)=-d \nu(z)$. The measure $\psi_{N}$ satisfies this symmetry, and therefore, the information obtained in this section can be used in the study of the frequency analysis problem as we do in the next section.

We consider the two special sequences of para-orthogonal polynomials $\left\{S_{n}(\nu, 1, z)\right\}$ and $\left\{S_{n}(\nu,-1, z)\right\}$. Since $S_{n}(\nu,-1, z)$ is divisible by $z-1$, we write

$$
R_{n}^{(1)}(\nu, z)=\frac{S_{n}(\nu, 1, z)}{1+S_{n}(\nu, 0)} \quad \text { and } \quad R_{n}^{(2)}(\nu, z)=\frac{S_{n+1}(\nu,-1, z)}{(z-1)\left(1-S_{n+1}(\nu, 0)\right)},
$$

for $n \geq 0$. The denominators are chosen in order to make the polynomials monic.

Clearly, $2 S_{n}(\nu, z)=\left(1+a_{n}^{(\nu)}\right) R_{n}^{(1)}(\nu, z)+\left(1-a_{n}^{(\nu)}\right)(z-1) R_{n-1}^{(2)}(\nu, z), n \geq 1$, which from (2) leads to

$$
2 z S_{n-1}(\nu, z)=R_{n}^{(1)}(\nu, z)+(z-1) R_{n-1}^{(2)}(\nu, z), \quad n \geq 1 .
$$

Theorem 3.1. The monic polynomials $R_{n}^{(\kappa)}(\kappa=1,2)$ satisfy $R_{0}^{(\kappa)}=1, R_{1}^{(\kappa)}(\nu, z)$ $=z+1$ and

$$
R_{n+1}^{(\kappa)}(\nu, z)=(z+1) R_{n}^{(\kappa)}(\nu, z)-4 \alpha_{n+1}^{(\kappa)} z R_{n-1}^{(\kappa)}(\nu, z), \quad n \geq 1,
$$

with $4 \alpha_{n+1}^{(1)}=\left(1+a_{n-1}^{(\nu)}\right)\left(1-a_{n}^{(\nu)}\right) \quad$ and $4 \alpha_{n+1}^{(2)}=\left(1-a_{n}^{(\nu)}\right)\left(1+a_{n+1}^{(\nu)}\right), \quad n \geq 1$.

The proof of this theorem is due to Delsarte and Genin [6].

The following theorem gives the relation between the Szegö polynomials $S_{n}$ and the orthogonal polynomials $\left\{P_{n}^{(\kappa)}\right\}$ that satisfy $P_{n}^{(\kappa)}(x(z))=(4 z)^{-n / 2} R_{n}^{(\kappa)}(\nu, z)$, through the transformation

$$
x(z)=\frac{1}{2}\left(z^{1 / 2}+z^{-1 / 2}\right) .
$$

\section{Theorem 3.2.}

1) Let $d \nu(z)$ be a positive measure on the unit circle such that the associated Szegö polynomials $\left\{S_{n}\right\}$ are all real (i.e., $-1<a_{n}^{(\nu)}<1$ for $n \geq 1$ ). Set

$\alpha_{n+1}^{(1)}=\frac{1}{4}\left(1+a_{n-1}^{(\nu)}\right)\left(1-a_{n}^{(\nu)}\right)>0 \quad$ and $\quad \alpha_{n+1}^{(2)}=\frac{1}{4}\left(1-a_{n}^{(\nu)}\right)\left(1+a_{n+1}^{(\nu)}\right)>0, \quad n \geq 1$ and define the positive measures $\phi^{(1)}$ and $\phi^{(2)}$ by

$$
d \phi^{(1)}(x)=-d \nu(z) \quad \text { and } \quad d \phi^{(2)}(x)=-\left(1-x^{2}\right) d \nu(z),
$$

where $x=x(z)=\frac{1}{2}\left(z^{1 / 2}+z^{-1 / 2}\right)$. The supports of $d \phi^{(1)}$ and $d \phi^{(2)}$ are inside $[-1,1]$.

Then (for $\kappa=1,2)$ the sequence of polynomials $\left\{P_{n}^{(\kappa)}\right\}$, given by $P_{0}^{(\kappa)}=$ $1, P_{1}^{(\kappa)}(x)=x$ and

$$
P_{n+1}^{(\kappa)}(x)=x P_{n}^{(\kappa)}(x)-\alpha_{n+1}^{(\kappa)} P_{n-1}^{(\kappa)}(x), \quad n \geq 1,
$$

is the sequence of monic orthogonal polynomials in relation to the measure $d \phi^{(\kappa)}$. 
2) Conversely, let $d \phi^{(1)}$ and $d \phi^{(2)}$ be two positive measures defined inside $[-1,1]$ such that $d \phi^{(2)}(x)=\left(1-x^{2}\right) d \phi^{(1)}(x)$. Let the respective monic orthogonal polynomials $P_{n}^{(1)}$ and $P_{n}^{(2)}$ associated with these measures satisfy $P_{n+1}^{(\kappa)}(x)=x P_{n}^{(\kappa)}(x)-\alpha_{n+1}^{(\kappa)} P_{n-1}^{(\kappa)}(x), n \geq 1$. Then the reflection coefficients $a_{n}^{(\nu)}$ of the Szegö polynomials $\left\{S_{n}\right\}$ associated with the positive measure $d \nu(z)=-d \phi^{(1)}(x(z))$ satisfy

$a_{n}^{(\nu)}=1-4 \alpha_{n+1}^{(1)} /\left(1+a_{n-1}^{(\nu)}\right) \quad$ and $\quad a_{n+1}^{(\nu)}=-1+4 \alpha_{n+1}^{(2)} /\left(1-a_{n}^{(\nu)}\right), \quad n \geq 1$, with $a_{0}^{(\nu)}=1$. Given explicitly (with $\mu_{0}^{(\kappa)}$ as the moment of order zero associated with $\left.\phi^{(\kappa)}\right)$,

$a_{2 n-1}^{(\nu)}=2 \frac{\alpha_{2 n-1}^{(2)} \alpha_{2 n-3}^{(2)} \cdots \alpha_{3}^{(2)} \mu_{0}^{(2)}}{\alpha_{2 n-1}^{(1)} \alpha_{2 n-3}^{(1)} \cdots \alpha_{3}^{(1)} \mu_{0}^{(1)}}-1 \quad$ and $\quad a_{2 n}^{(\nu)}=2 \frac{\alpha_{2 n}^{(2)} \alpha_{2 n-2}^{(2)} \cdots \alpha_{2}^{(2)}}{\alpha_{2 n}^{(1)} \alpha_{2 n-2}^{(1)} \cdots \alpha_{2}^{(1)}}-1, \quad n \geq 1$

Moreover,

$$
2 z S_{n-1}(\nu, z)=R_{n}^{(1)}(\nu, z)+(z-1) R_{n-1}^{(2)}(\nu, z), \quad n \geq 1,
$$

where $R_{n}^{(\kappa)}(\nu, z)=(4 z)^{n / 2} P_{n}^{(\kappa)}(x(z))$.

This theorem follows from results given in [3] and [22].

One can also show that (see [2])

$$
P_{n}^{(1)}(x)=P_{n}^{(2)}(x)-\frac{1}{4}\left(1-a_{n}^{(\nu)}\right)\left(1-a_{n-1}^{(\nu)}\right) P_{n-2}^{(2)}(x), \quad n \geq 1,
$$

and

$$
P_{n}^{(1)}(x)=x P_{n-1}^{(2)}(x)-\frac{1}{2}\left(1-a_{n-1}^{(\nu)}\right) P_{n-2}^{(2)}(x), \quad n \geq 1
$$

This last equation leads to the following.

Theorem 3.3. The $n-1$ zeros of the polynomial $P_{n-1}^{(2)}$ and the $n$ zeros of the polynomial $P_{n}^{(1)}$ interlace.

Now we state some results on the associated quadrature formulas.

Theorem 3.4. Let the positive measure $\nu$ on the unit circle and the positive measures $\phi^{(1)}$ and $\phi^{(2)}$ on $[-1,1]$ be such that

$$
-d \nu(z)=d \phi^{(1)}(x(z))=\left(1-x(z)^{2}\right)^{-1} d \phi^{(2)}(x(z))
$$

with $x(z)=\left(z^{1 / 2}+z^{-1 / 2}\right) / 2$. That is,

$$
-d \nu\left(e^{i \theta}\right)=d \phi^{(1)}(\cos (\theta / 2))=\left(\sin ^{2}(\theta / 2)\right)^{-1} d \phi^{(2)}(\cos (\theta / 2)) .
$$

Let

$R L Q R(\kappa): \quad \int_{-1}^{1} g(x) d \phi^{(\kappa)}(x)=\sum_{m=1}^{n} \chi_{m, n}^{(\kappa)} g\left(x_{m, n}^{(\kappa)}\right), \quad(\kappa=1,2)$,

be the $n$-point Gaussian quadrature rule based on the zeros of $P_{n}^{(\kappa)}(x)$. The nodes $x_{m, n}^{(\kappa)}$ are arranged in the order $1>x_{1, n}^{(\kappa)}>x_{2, n}^{(\kappa)}>\cdots>x_{n, n}^{(\kappa)}>-1$.

Let

$\operatorname{UCQR}(\tau): \quad \int_{0}^{2 \pi} f\left(e^{i \theta}\right) d \nu\left(e^{i \theta}\right)=\sum_{m=1}^{n} \lambda_{m, n}(\nu, \tau) f\left(z_{m, n}(\nu, \tau)\right), \quad(\tau= \pm 1)$ 
be the quadrature rule (4) of order $n$, with $\tau=1$ or -1 , which is exact for $f \in$ $\Omega_{n-1}$. The nodes $z_{m, n}(\nu, \tau)=e^{i \theta_{m, n}(\nu, \tau)}$ are arranged in the order $0<\theta_{1, n}(\nu, \tau)<$ $\theta_{2, n}(\nu, \tau)<\cdots<\theta_{n, n}(\nu, \tau) \leq 2 \pi$.

Then the following relations hold.

(1) $x_{m, n}^{(1)}=\cos \left(\frac{1}{2} \theta_{m, n}(\nu, 1)\right), \chi_{m, n}^{(1)}=\lambda_{m, n}(\nu, 1)$, for $m=1,2, \ldots, n$.

(2) $x_{m, n}^{(2)}=\cos \left(\frac{1}{2} \theta_{m, n+1}(\nu,-1)\right), \chi_{m, n}^{(2)}=\sin ^{2}\left(\frac{1}{2} \theta_{m, n+1}(\nu,-1)\right) \lambda_{m, n+1}(\nu,-1)$, for $m=1,2, \ldots, n$, and $z_{n+1, n+1}(\nu,-1)=e^{i 2 \pi}=1, \lambda_{n+1, n+1}(\nu,-1)$ $=\mu_{0}^{(\nu)}-\sum_{m=1}^{n} \lambda_{m, n+1}(\nu,-1)$.

Proof. First, $R L Q R$ stands for Real Line Quadrature Rule and $U C Q R$ stands for Unit Circle Quadrature Rule.

The proof of the first part of this theorem that relates $R L Q R(1)$ and $U C Q R(1)$ can be obtained from [4, Theorem 3.1].

To obtain the second part of the theorem that relates $R L Q R(2)$ and $U C Q R(-1)$, we first note that $x_{m, n}^{(2)}$ are the zeros of $P_{n}^{(2)}(x)$ and $z_{m, n+1}(\nu,-1)(1 \leq m \leq n)$ are the zeros of $R_{n}^{(2)}(\nu, z)=(4 z)^{n / 2} P_{n}^{(2)}(x(z))$, which satisfy

$$
R_{n}^{(2)}(\nu, z)=\frac{S_{n+1}(\nu,-1, z)}{(z-1)\left(1-S_{n+1}(\nu, 0)\right)} .
$$

If $f(z) \in \Omega_{n-1}$, then $f(z)(z-1)^{2} / z \in \Omega_{n}$. Hence by substitution of $f(z)(z-1)^{2} / z$ in $U C Q R(-1)$ of order $n+1$, we obtain

$$
\begin{aligned}
\int_{0}^{2 \pi} & {\left[-4 \sin ^{2}\left(\frac{1}{2} \theta\right)\right] f\left(e^{i \theta}\right) d \nu\left(e^{i \theta}\right) } \\
& =\sum_{m=1}^{n} \lambda_{m, n+1}(\nu,-1)\left[-4 \sin ^{2}\left(\frac{1}{2} \theta_{m, n+1}(\nu,-1)\right)\right] f\left(z_{m, n+1}(\nu,-1)\right),
\end{aligned}
$$

for $f \in \Omega_{n-1}$. On the other hand, by application of the first part of this theorem to the measure $-\left(1-\cos ^{2}(\theta / 2)\right) d \nu\left(e^{i \theta}\right)=d \phi^{(2)}(\cos (\theta / 2))$, we obtain

$$
\int_{0}^{2 \pi}\left[\sin ^{2}\left(\frac{1}{2} \theta\right)\right] f\left(e^{i \theta}\right) d \nu\left(e^{i \theta}\right)=\sum_{m=1}^{n} \chi_{m, n}^{(2)} f\left(z_{m, n+1}(\nu,-1)\right),
$$

which is exact whenever $f(z) \in \Omega_{n-1}$. Hence comparing the above two quadrature rules, we obtain

$$
\chi_{m, n}^{(2)}=\sin ^{2}\left(\frac{1}{2} \theta_{m, n+1}(\nu,-1)\right) \lambda_{m, n+1}(\nu,-1), \quad m=1,2, \ldots, n .
$$

To determine the value of $\lambda_{n+1, n+1}(\nu,-1)$ associated with the node $z_{n+1, n+1}(\nu,-1)$ $=1$, we use

$$
\mu_{0}^{(\nu)}=\sum_{m=1}^{n+1} \lambda_{m, n+1}(\nu,-1) .
$$

This completes the proof of the theorem.

Numerical Evaluation. The polynomials $P_{n}^{(\kappa)}$ associated with the measure $\phi^{(\kappa)}$, more precisely the coefficients $\alpha_{n}^{(\kappa)}$, can also be evaluated directly from the moments of the measure $\nu(z)$. For this we require the following algorithm known as the modified Chebyshev algorithm.

Let $\phi$ be a positive measure on $\mathbb{R}$ and let the so-called modified moments $\hat{\mu}_{l}^{(\phi)}=$ $\int_{\mathbb{R}} Q_{l}(x) d \phi(x), l=0,1, \ldots, 2 n-1$, be known. Here, the polynomials $Q_{l}$ are given by the recurrence relation

$$
Q_{l+1}(x)=\left(x-\hat{\beta}_{l+1}\right) Q_{l}(x)-\hat{\alpha}_{l+1} Q_{l-1}(x), \quad l=1,2, \ldots, 2 n-2,
$$


with $Q_{0}=1$ and $Q_{1}(x)=x-\hat{\beta}_{1}$, with the numbers $\hat{\beta}_{l}$ and $\hat{\alpha}_{l}$ chosen arbitrarily.

Note that if one chooses $\hat{\beta}_{l}=\hat{\alpha}_{l+1}=0, l=1,2, \ldots$, then the $\int_{\mathbb{R}} Q_{l}(x) d \phi(x)$ are the ordinary moments $\int_{\mathbb{R}} x^{l} d \phi(x)$.

The algorithm can be given as

Algorithm II (Modified Chebyshev algorithm).

Set

$c_{-1, l}=0, \quad c_{0, l}=\hat{\mu}_{l}^{(\phi)}, \quad l=0,1, \ldots, 2 n-1$.

$\beta_{1}=\hat{\beta}_{1}+c_{0,1} / c_{0,0}$.

For $m=1,2, \ldots, n-1, \mathbf{d o}$

$$
\begin{aligned}
& c_{m, l}=c_{m-1, l+2}+\left(\hat{\beta}_{m+l+1}-\beta_{m}\right) c_{m-1, l+1}-\alpha_{m} c_{m-2, l+2} \\
& +\hat{\alpha}_{m+l+1} c_{m-1, l}, \quad l=0,1, \ldots, 2 n-2 m-1, \\
& \beta_{m+1}=\hat{\beta}_{m+1}+\frac{c_{m, 1}}{c_{m, 0}}-\frac{c_{m-1,1}}{c_{m-1,0}}, \quad \alpha_{m+1}=\frac{c_{m, 0}}{c_{m-1,0}} .
\end{aligned}
$$

The above algorithm, first presented by Sack and Donovan [18, determines the coefficients in the three term recurrence relation

$$
P_{m+1}(x)=\left(x-\beta_{m+1}\right) P_{m}(x)-\alpha_{m+1} P_{m-1}(x), \quad m=1,2, \ldots, n-1,
$$

where $P_{0}(x)=1, P_{1}(x)=x-\beta_{1}$, of the orthogonal polynomials $\left\{P_{n}\right\}_{m=0}^{n}$ associated with the measure $\phi$. In practice (see Gautschi [8]), a good choice of the numbers $\hat{\beta}_{l}$ and $\hat{\alpha}_{l}$ makes the algorithm numerically more stable.

We now see how this algorithm can be used to determine the coefficients $\alpha_{n}^{(1)}$ associated with the measure $\phi^{(1)}$. Note that $\beta_{n}^{(1)}$ are all equal to zero.

Since $d \nu\left(e^{i \theta}\right)$ is a symmetric measure,

$$
\mu_{m}^{(\nu)}=\int_{0}^{2 \pi} e^{i m \theta} d \nu\left(e^{i \theta}\right)=\int_{0}^{2 \pi} \cos (m \theta) d \nu\left(e^{i \theta}\right) .
$$

Hence using $d \nu\left(e^{i \theta}\right)=-d \phi^{(1)}(\cos (\theta / 2))$ and $x=\cos (\theta / 2)$, we obtain

$$
\mu_{m}^{(\nu)}=\int_{-1}^{1} T_{2 m}(x) d \phi^{(1)}(x), \quad m \geq 0,
$$

where the $T_{m}$ are the $m$-th degree Chebyshev polynomials of the first kind. Hence using the monic Chebyshev polynomials $\hat{T}_{m}=2^{-m+1} T_{m}, m \geq 1$, we can define the modified moments

$$
\hat{\mu}_{0}^{\left(\phi^{(1)}\right)}=\mu_{0}^{(\nu)}, \quad \hat{\mu}_{2 m-1}^{\left(\phi^{(1)}\right)}=0, \quad \hat{\mu}_{2 m}^{\left(\phi^{(1)}\right)}=2^{-2 m+1} \mu_{m}^{(\nu)}, \quad m \geq 1,
$$

and apply the modified Chebyshev algorithm to obtain the coefficients $\alpha_{n}^{(1)}$. Since

$$
\begin{aligned}
& \hat{T}_{1}(x)=x, \quad \hat{T}_{2}(x)=x \hat{T}_{m}(x)-\frac{1}{2} \hat{T}_{0}(x), \\
& \hat{T}_{m+1}(x)=x \hat{T}_{m}(x)-\frac{1}{4} \hat{T}_{m-1}(x), \quad m=2,3, \ldots, 2 n-2,
\end{aligned}
$$

and since the $c_{m, 2 l+1}$ turn out to be zero for all $m$, letting $d_{0,0}=c_{0,0}$ and $d_{m, l}=$ $2^{2(m+l)-1} c_{m, 2 l}$, we obtain the algorithm 
Algorithm III.

Set

$$
\begin{aligned}
& d_{0, l}=\mu_{l}^{(\nu)}=\int_{0}^{2 \pi} e^{i l \theta} d \nu\left(e^{i \theta}\right), \quad l=0,1, \ldots, n-1 . \\
& d_{1, l}=d_{0, l+1}+d_{0, l}, \quad l=0,1, \ldots, n-2, \\
& \hat{d}_{2}=2 \frac{d_{1,0}}{d_{0,0}} .
\end{aligned}
$$

For $m=2,3, \ldots, n-1$, do

$$
\begin{aligned}
& d_{m, l}=d_{m-1, l+1}+d_{m-1, l}-\hat{d}_{m} d_{m-2, l+1}, \quad l=0,1, \ldots, n-m-1, \\
& \hat{d}_{m+1}=\frac{d_{m, 0}}{d_{m-1,0}} .
\end{aligned}
$$

From this algorithm

$$
\alpha_{m}^{(1)}=\frac{1}{4} \hat{d}_{m}, \quad m=2,3, \ldots, n-1 .
$$

Having found the values of $\alpha_{m}^{(1)}$, if needed, the values of $\alpha_{m}^{(2)}$ can be generated by

$$
\alpha_{m+1}^{(2)}=\frac{1}{4}\left(1-a_{m}^{(\nu)}\right)\left(1+a_{m+1}^{(\nu)}\right), \quad m \geq 1,
$$

where $a_{1}^{(\nu)}=1-\hat{d}_{2} / 2$ and $a_{m}^{(\nu)}=1-\hat{d}_{m+1} /\left(1+a_{m-1}^{(\nu)}\right), m \geq 2$, are the reflection coefficients of the Szegő polynomials associated with $\nu$.

\section{REAL ORTHOGONAL POLYNOMIALS IN FREQUENCY ANALYSIS}

In this section, we apply the results obtained in the previous sections to the frequency analysis problem.

For $\kappa=1,2$, consider the monic polynomials $P_{m}^{(\kappa)}(N, x), m=1,2, \ldots$, which satisfy the recurrence relation

$$
P_{m+1}^{(\kappa)}(N, x)=x P_{m}^{(\kappa)}(N, x)-\alpha_{m+1}^{(\kappa)}(N) P_{m-1}^{(\kappa)}(N, x), \quad m \geq 1,
$$

with $P_{0}^{(\kappa)}(N, x)=1, P_{1}^{(\kappa)}(N, x)=x$ and

$$
\alpha_{m+1}^{(1)}(N)=\frac{1}{4}\left(1+a_{m-1}^{(N)}\right)\left(1-a_{m}^{(N)}\right) \quad \text { and } \quad \alpha_{m+1}^{(2)}(N)=\frac{1}{4}\left(1-a_{m}^{(N)}\right)\left(1+a_{m+1}^{(N)}\right) .
$$

Here $a_{m}^{(N)}=S_{m}\left(\psi_{N}, 0\right)$. Then Theorem 3.2 tells us that $P_{m}^{(\kappa)}(N, x), m=0,1, \ldots$, are the monic orthogonal polynomials associated with the positive measure $\phi^{(\kappa)}(N, x)$ on $[-1,1]$, where

$$
d \phi^{(1)}(N, \cos (\theta / 2))=[\sin (\theta / 2)]^{-2} d \phi^{(2)}(N, \cos (\theta / 2))=-d \psi_{N}\left(e^{i \theta}\right) .
$$

Furthermore,

$$
P_{m}^{(\kappa)}(N, x(z))=(4 z)^{-m / 2} R_{m}^{(\kappa)}\left(\psi_{N}, z\right),
$$

where $x(z)=\left(z^{1 / 2}+z^{-1 / 2}\right) / 2$. Let

$$
\int_{-1}^{1} g(x) d \phi^{(\kappa)}(N, x)=\sum_{j=1}^{n} \chi_{j, n}^{(\kappa)}(N) g\left(x_{j, n}^{(\kappa)}(N)\right)
$$

be the Gaussian rule on the zeros of $P_{n}^{(\kappa)}(N, x)$. Then from (18) and from Theorem [3.4, we have the following. 
(A) The information obtained from the behavior of the zeros

$$
z_{m, n}(N, 1)=e^{i \theta_{m, n}(N, 1)}
$$

and quadrature weights $\lambda_{m, n}(N, 1)$ associated with $S_{n}\left(\psi_{N}, 1, z\right)$ is just the same as that obtained from the behavior of the zeros $x_{m, n}^{(1)}(N)=$ $\cos \left(\frac{1}{2} \theta_{m, n}(N, 1)\right)$ and quadrature weights of $\chi_{m, n}^{(1)}(N)=\lambda_{m, n}(N, 1)$ associated with $P_{n}^{(1)}(N, x)$.

(B) Likewise, the information obtained from the behavior of the zeros

$$
z_{m, n}(N,-1)=e^{i \theta_{m, n}(N,-1)} \text { of }(z-1)^{-1} S_{n}\left(\psi_{N},-1, z\right)
$$

is just the same as that obtained from the behavior of the zeros $x_{m, n-1}^{(2)}(N)=$ $\cos \left(\frac{1}{2} \theta_{m, n}(N,-1)\right)$ of $P_{n-1}^{(2)}(N, x)$.

Thus, we consider the measure $\phi$ given by

$$
d \phi(\cos (\theta / 2))=-d \psi\left(e^{i \theta}\right),
$$

where $\psi$ is given by (3). We can write

$$
d \phi(x)=\sum_{k=1}^{n_{0}} \lambda_{k} H\left(x-\xi_{k}\right)
$$

where $\xi_{k}=\cos \omega_{k}$ and $\lambda_{k}$ are as in (3). Note that the $\xi_{k}$ are ordered as

$$
-1<\xi_{n_{0}}<\cdots<\xi_{2}<\xi_{1}<1 .
$$

Thus it is natural to order the zeros of $P_{n}^{(\kappa)}(N, x)$ as

$$
-1<x_{n, n}^{(\kappa)}(N)<\cdots<x_{2, n}^{(\kappa)}(N)<x_{1, n}^{(\kappa)}(N)<1 .
$$

The following theorem gives some information on the asymptotic behavior of the measure $\phi^{(1)}(N, x)$ and the asymptotic behavior of the polynomials $P_{n}^{(1)}(N, x)$ when $1 \leq n \leq n_{0}$.

Theorem 4.1. We have

$$
\lim _{N \rightarrow \infty} \int_{-1}^{1} g(x) d \phi^{(1)}(N, x)=\int_{-1}^{1} g(x) d \phi(x),
$$

for any $g$ continuous on $[-1,1]$.

(2) For each fixed $n, 1 \leq n \leq n_{0}$,

$$
\lim _{N \rightarrow \infty} P_{n}^{(1)}(N, x)=P_{n}(\phi, x), \quad x \in[-1,1],
$$

where $P_{n}(\phi, x)$ are the orthogonal polynomials associated with the discrete measure $\phi$. In particular,

$$
\lim _{N \rightarrow \infty} P_{n_{0}}^{(1)}(N, x)=P_{n_{0}}(\phi, x)=\prod_{m=1}^{n_{0}}\left(x-\xi_{m}\right), \quad x \in[-1,1],
$$

where $\xi_{m}=\cos \left(\frac{1}{2} \omega_{m}\right)$.

Proof. The proof of this theorem follows from Theorem A and from equations (11) and (12).

The next theorem gives some information on the zeros and Christoffel numbers associated with $P_{n}^{(1)}(N, x)$. This information can be used to detect the frequencies and the modulus of the amplitudes of the given signal. 
Theorem 4.2. Let $n \geq n_{0}$ be fixed.

(1) Then for any $\epsilon>0$ there exists an $N(\epsilon)$ such that for all $N \geq N(\epsilon)$, each of the intervals $\left(\xi_{m}-\epsilon, \xi_{m}+\epsilon\right), m=1,2, \ldots, n_{0}$, contains at least one zero of $P_{n}^{(1)}(N, x)$.

(2) Let $\epsilon>0$ be such that the intervals $\Delta_{j}(\epsilon)=\left(\xi_{j}-\epsilon, \xi_{j}+\epsilon\right), j=1,2, \ldots, n_{0}$, satisfy

$$
\Delta_{j}(\epsilon) \subset(-1,1) \quad \text { and } \quad \xi_{k} \notin \Delta_{j}(\epsilon) \text { if } k \neq j .
$$

Set $\hat{\Delta}(\epsilon)=[0,2 \pi] \backslash \bigcup_{j=1}^{n_{0}} \Delta_{j}(\epsilon)$. Then

$$
\begin{aligned}
& \lim _{N \rightarrow \infty} \sum_{x_{k, n}^{(1)}(N) \in \Delta_{j}(\epsilon)} \chi_{k, n}^{(1)}(N, \tau)=\lambda_{j}, \quad j=1,2, \ldots, n_{0}, \\
& \lim _{N \rightarrow \infty} \sum_{x_{k, n}^{(1)}(N) \in \hat{\Delta}(\epsilon)} \chi_{k, n}^{(1)}(N, \tau)=0 .
\end{aligned}
$$

Proof. The proof of this theorem is a consequence of Theorem 2.2, Corollary 2.4 and of the equations

$$
\xi_{m}=\cos \left(\frac{1}{2} \omega_{m}\right), \quad x_{m, n}^{(1)}(N)=\cos \left(\frac{1}{2} \theta_{m, n}(N, 1)\right) \quad \text { and } \quad \chi_{m, n}^{(1)}(N)=\lambda_{m, n}(N, 1),
$$

which follow from results obtained in Section 3.

The first part of the above theorem tells us that, for each $N$, we can pick out a zero, let us say $x_{m}(N)\left(m=1,2, \ldots, n_{0}\right)$, of $P_{n}^{(1)}(N, x)$ such that the sequence $\left\{x_{m}(N)\right\}_{N=1}^{\infty}$ has the limit $\xi_{m}$.

Now we have the following theorem on distinct convergent sequences of zeros.

Theorem 4.3. Let $n>n_{0}$ be fixed. Let $\Lambda$ be a subsequence of the sequence of natural numbers and, for each $N \in \Lambda$, let $y(1, N)>y(2, N)>y(3, N)$ be three distinct zeros of $P_{n}^{(1)}(N, x)$ such that the limits

$$
\lim _{\substack{N \rightarrow \infty \\ N \in \Lambda}} y(j, N)=y(j), \quad j=1,2,3,
$$

all exist. Then the following must hold.

(1) It is not possible to have $y(1)=y(2)=y(3)$. That is, three distinct convergent subsequences of zeros cannot have a common limit.

(2) If $y(1)=y(2)$, that is, if two distinct convergent subsequences of zeros have a common limit, then this limit must be equal to one of the points $\xi_{m}$, $m=1,2, \ldots, n_{0}$.

Proof. With $x(z)=\left(z^{1 / 2}+z^{-1 / 2}\right) / 2$, since $P_{n}^{(1)}(N, x(z))=(4 z)^{-n / 2} \frac{S_{n}\left(\psi_{N}, 1, z\right)}{1+a_{n}^{(N)}}$ and $P_{n-1}^{(2)}(N, x(z))=(4 z)^{-(n-1) / 2} \frac{S_{n}\left(\psi_{N},-1, z\right)}{(z-1)\left(1-a_{n}^{(N)}\right)}$, we obtain from Theorem 2.5 that there exists a subsequence $\Lambda_{2}$ of $\Lambda$ such that

$$
\begin{aligned}
& \lim _{\substack{N \rightarrow \infty \\
N \in \Lambda_{2}}} P_{n}^{(1)}(N, x)=Q_{n-n_{0}}^{(1)}(x) \prod_{m=0}^{n_{0}}\left(x-\xi_{m}\right), \\
& \lim _{\substack{N \rightarrow \infty \\
N \in \Lambda_{2}}} P_{n-1}^{(2)}(N, x)=Q_{n-n_{0}-1}^{(2)}(x) \prod_{m=0}^{n_{0}}\left(x-\xi_{m}\right),
\end{aligned}
$$

where $Q_{n-n_{0}}^{(1)}$ and $Q_{n-n_{0}-1}^{(2)}$ do not have any common zeros. 
Now we need to use the interlacing property of the zeros of $P_{n}^{(1)}(N, x)$ and $P_{n-1}^{(2)}(N, x)$, which follows from Theorem 3.3

Suppose that $y(1)=y(2)=y$. From the interlacing properties of the zeros we find that there exists a zero $\hat{y}(1, N)$ of $P_{n-1}^{(2)}(N, x)$ such that $y(1, N)>\hat{y}(1, N)>$ $y(2, N)$ and hence,

$$
\lim _{\substack{N \rightarrow \infty \\ N \in \Lambda}} y(1, N)=\lim _{\substack{N \in \infty \\ N \in \Lambda}} \hat{y}(1, N)=y .
$$

Hence part (2) of the theorem follows from (13).

Now suppose that $y(1)=y(2)=y(3)=y$. From the interlacing property of the zeros there exist two zeros $\hat{y}(1, N)$ and $\hat{y}(2, N)$ of $P_{n-1}^{(2)}(N, x)$ such that $y(1, N)>\hat{y}(1, N)>y(2, N)>\hat{y}(2, N)>y(3, N)$ and hence,

$$
\lim _{\substack{N \rightarrow \infty \\ N \in \Lambda}} y(1, N)=\lim _{\substack{N \rightarrow \infty \\ N \in \Lambda}} \hat{y}(1, N)=\lim _{\substack{N \rightarrow \infty \\ N \in \Lambda}} y(2, N)=\lim _{\substack{N \rightarrow \infty \\ N \in \Lambda}} \hat{y}(2, N)=y .
$$

This is a contradiction to the fact that $Q_{n-n_{0}}^{(1)}$ and $Q_{n-n_{0}-1}^{(2)}$ do not have any common zeros. This completes part (1) of the theorem.

We now propose the following procedure to solve the frequency analysis problem. The procedure is divided into three steps.

Step 1. Determine the coefficients $\alpha_{m}^{(1)}(N), m=2,3, \ldots, n$, in the recurrence relation (10). This can be done by the special version of the modified Chebyshev algorithm:

$$
\begin{aligned}
& \text { Algorithm IV. } \\
& \text { Set } \\
& \qquad \begin{array}{l}
d_{0, l}=\mu_{l}^{(N)}=\frac{1}{N} \sum_{m=l}^{N-1} x(m) x(m-l), \quad l=0,1, \ldots, n-1 . \\
d_{1, l}=d_{0, l+1}+d_{0, l}, \quad l=0,1, \ldots, n-2, \\
\hat{d}_{2}=2 \frac{d_{1,0}}{d_{0,0}}
\end{array} \\
& \text { For } m=2,3, \ldots, n-1, \text { do } \\
& \qquad \begin{array}{l}
d_{m, l}=d_{m-1, l+1}+d_{m-1, l}-\hat{d}_{m} d_{m-2, l+1}, \quad l=0,1, \ldots, n-m-1, \\
\hat{d}_{m+1}=\frac{d_{m, 0}}{d_{m-1,0}} .
\end{array}
\end{aligned}
$$

From this

$$
\alpha_{m}^{(1)}(N)=\frac{1}{4} \hat{d}_{m}, \quad m=2,3, \ldots, n .
$$

Remarks. (1) The reflection coefficients associated with the Szegö polynomials $S_{m}\left(\psi_{N}, z\right)$ can be derived from

$$
a_{m}^{(N)}=1-\hat{d}_{m+1} /\left(1+a_{m-1}^{(N)}\right), \quad \text { for } \quad m=1,2, \ldots, n-1,
$$

with $a_{0}^{(N)}=1$.

(2) The Levinson algorithm requires in the order of $n^{2}-4$ multiplications to obtain $\left\{a_{m}^{(N)}\right\}_{m=1}^{n-1}$, while the above algorithm requires in the order of $\frac{1}{2} n^{2}+\frac{1}{2} n-1$ multiplications. This means that the above algorithm is about twice the speed of 
the Levinson algorithm. The above algorithm is in some sense a modification of the split Levinson algorithm (see [7]).

(3) Furthermore, the coefficients $\alpha_{m}^{(2)}(N)$ can be generated by

$$
\alpha_{m}^{(2)}(N)=\frac{1}{4}\left(1-a_{m-1}^{(N)}\right)\left(1+a_{m}^{(N)}\right), \quad \text { for } \quad m=2,3, \ldots, n-1 .
$$

Step 2. Determine the zeros $x_{m, n}^{(1)}(N)$ and the Christoffel numbers $\chi_{m, n}^{(1)}(N)$ associated with the orthogonal polynomials $P_{n}^{(1)}(N, x)$. These can be generated as the eigenvalue problem associated with the symmetric Jacobi matrix $J_{n}(d)$

$$
\left[\begin{array}{cccccc}
d & \sqrt{\alpha_{2}^{(1)}(N)} & 0 & \cdots & 0 & 0 \\
\sqrt{\alpha_{2}^{(1)}(N)} & d & \sqrt{\alpha_{3}^{(1)}(N)} & \cdots & 0 & 0 \\
0 & \sqrt{\alpha_{3}^{(1)}(N)} & d & \cdots & 0 & 0 \\
\vdots & \vdots & \vdots & & \vdots & \vdots \\
0 & 0 & 0 & \cdots & d & \sqrt{\alpha_{n}^{(1)}(N)} \\
0 & 0 & 0 & \cdots & \sqrt{\alpha_{n}^{(1)}(N)} & d
\end{array}\right] .
$$

The eigenvalues of $J_{n}(d)$ are $d+x_{m, n}^{(1)}(N), m=1,2, \ldots, n$, and if $\eta_{m}$ is the normalized $\left(\eta_{m}^{T} \eta_{m}=1\right)$ eigenvector associated with the eigenvalue $d+$ $x_{m, n}^{(1)}(N)$, then

$$
\chi_{m, n}^{(1)}(N)=\left(\eta_{m, 1}\right)^{2} \mu_{0}^{(N)} .
$$

Here, $\eta_{m, 1}$ is the first component of $\eta_{m}$.

Step 3. Finally, using the results given in the Theorems 4.114.2 and 4.3, determine the number of frequencies $n_{0}$, the frequencies $\omega_{m}$ and the modulus of the amplitudes $\gamma_{m}$, by observing the limiting behavior of $x_{m, n}^{(1)}(N)$ and $\chi_{m, n}^{(1)}(N)$ from growing values of $N$.

\section{EXAMPLes}

We give some examples to show that the method proposed in the previous section is highly feasible. All the calculations are performed using the software Maple.

Example 1. We consider the signal

$$
x(m)=2 e^{i m \pi}+(1+2 i) e^{i 2 m \pi / 3}+(1-2 i) e^{i 4 m \pi / 3}+Z_{m},
$$

embedded with a random perturbation (or noise) $Z_{m}$ at each discrete time $m=$ $0,1,$. We choose $Z_{m}$ to be real random numbers in the range [-0.005, 0.005].

We expect that the interesting zeros of $P_{n}^{(1)}(N, x)$, as $N$ tends to infinity, converge to the values

$$
\xi_{1}=\cos (2 \pi / 6)=0.5, \quad \xi_{2}=\cos (\pi / 2)=0, \quad \text { and } \quad \xi_{3}=\cos (4 \pi / 6)=-0.5 .
$$

The corresponding quadrature weights (or sums of quadrature weights as in Theorem 4.2) tend to the limits

$$
\lambda_{1}=5, \quad \lambda_{2}=4, \quad \text { and } \quad \lambda_{3}=5 .
$$


TABLE 1. The nonnegative zeros of $P_{n}^{(1)}(N, x)$, for $n=7$, and the corresponding quadrature weights for the signal given by (14).

\begin{tabular}{|c|c|c|c|c|}
\hline$N$ & 1000 & 10000 & 100000 & 1000000 \\
\hline$x_{1, n}^{(1)}(N)$ & .9594011810 & .9594358752 & .9600074510 & .9638104402 \\
$x_{2, n}^{(1)}(N)$ & .6240716070 & .6239469630 & .6262791465 & .6463467115 \\
$x_{3, n}^{(1)}(N)$ & .4993490519 & .4999350137 & .4999935864 & .4999994190 \\
$x_{4, n}^{(1)}(N)$ & .0000000000 & .0000000000 & .0000000000 & .0000000000 \\
$\chi_{1, n}^{(1)}(N)$ & $.2385586 e-2$ & $.2398284 e-3$ & $.2510096 e-4$ & $.3672402 e-5$ \\
$\chi_{2, n}^{(1)}(N)$ & $.3019255 e-1$ & $.3038765 e-2$ & $.2968618 e-3$ & $.2512087 e-4$ \\
$\chi_{3, n}^{(1)}(N)$ & $.4960261 e+1$ & $.4995888 e+1$ & $.4999684 e+1$ & $.4999974 e+1$ \\
$\chi_{4, n}^{(1)}(N)$ & $.3980690 e+1$ & $.3998053 e+1$ & $.3999756 e+1$ & $.3999974 e+1$ \\
\hline
\end{tabular}

We calculate the zeros of $P_{7}^{(1)}(N, x)$ and $P_{16}^{(1)}(N, x)$ to analyze their behavior. Because of the symmetry in the zeros of $P_{n}^{(1)}(N, x)$, we give, respectively in Table 1 and Table 2 only the nonnegative zeros of $P_{7}^{(1)}(N, x)$ and $P_{16}^{(1)}(N, x)$ and the corresponding quadrature weights. As one can see from Table 1, as $N$ gets larger, $\chi_{1,7}^{(1)}(N)$ and $\chi_{2,7}^{(1)}(N)$ tend to the limit 0 and hence, $x_{1,7}^{(1)}(N)$ and $x_{2,7}^{(1)}(N)$ represent the uninteresting zeros. The other two zeros, which are interesting ones, permit us to recover the limits $\xi_{1}=0.5$ and $\xi_{2}=0$. The corresponding quadrature weights also tend to the limits $\lambda_{1}=5$ and $\lambda_{2}=4$.

In Table 2. the degree of the polynomial is even. As we can see, this forces the zero $x_{8,16}^{(1)}(N)$ (and also the zero $x_{9,16}^{(1)}(N)$ ) to the limit $\xi_{2}=0$. According to Theorem 4.3. convergence of these two zeros to $\xi_{2}$ means no other zeros can

TABle 2. Positive zeros of $P_{n}^{(1)}(N, x)$, for $n=16$, and the corresponding quadrature weights for the signal given by (14).

\begin{tabular}{|c|c|c|c|c|}
\hline$N$ & 1000 & 10000 & 100000 & 1000000 \\
\hline$x_{1, n}^{(1)}(N)$ & .9940844167 & .9940870898 & .9941331278 & .9943640409 \\
$x_{2, n}^{(1)}(N)$ & .9472732272 & .9473005082 & .9475947640 & .9495377568 \\
$x_{3, n}^{(1)}(N)$ & .8548244375 & .8548903013 & .8555067037 & .8603075329 \\
$x_{4, n}^{(1)}(N)$ & .7189582997 & .7189420977 & .7197975661 & .7268018340 \\
$x_{5, n}^{(1)}(N)$ & .5385688762 & .5380815523 & .5386536378 & .5438509641 \\
$x_{6, n}^{(1)}(N)$ & .4993173295 & .4999312006 & .4999932243 & .4999994002 \\
$x_{7, n}^{(1)}(N)$ & .2904740721 & .2904681641 & .2906274219 & .2920830261 \\
$x_{8, n}^{(1)}(N)$ & $.6701693 e-2$ & $.2113208 e-2$ & $.6692490 e-3$ & $.2156445 e-3$ \\
$\chi_{1, n}^{(1)}(N)$ & $.7977139 e-3$ & $.8018650 e-4$ & $.8492834 e-5$ & $.1341455 e-5$ \\
$\chi_{2, n}^{(1)}(N)$ & $.9460595 e-3$ & $.9528461 e-4$ & $.9991095 e-5$ & $.1489447 e-5$ \\
$\chi_{3, n}^{(1)}(N)$ & $.1415432 e-2$ & $.1419179 e-3$ & $.1466860 e-4$ & $.1942683 e-5$ \\
$\chi_{4, n}^{(1)}(N)$ & $.3392576 e-2$ & $.3401662 e-3$ & $.3437371 e-4$ & $.3808523 e-5$ \\
$\chi_{5, n}^{(1)}(N)$ & $.9917225 e-1$ & $.1030305 e-1$ & $.1005570 e-2$ & $.8145786 e-4$ \\
$\chi_{6, n}^{(1)}(N)$ & $.4881227 e+1$ & $.4987618 e+1$ & $.4998873 e+1$ & $.4999906 e+1$ \\
$\chi_{7, n}^{(1)}(N)$ & $.6356956 e-2$ & $.6361711 e-3$ & $.6434639 e-4$ & $.7037543 e-5$ \\
$\chi_{8, n}^{(1)}(N)$ & $.1989875 e+1$ & $.1998979 e+1$ & $.1999873 e+1$ & $.1999986 e+1$ \\
\hline
\end{tabular}


converge to the same limit. Note that, as indicated by Theorem 4.2, the sum $\chi_{8,16}^{(1)}(N)+\chi_{9,16}^{(1)}(N)$ converges to the limit $\lambda_{2}=4$.

From the quadrature weights, the only other interesting zero of $P_{16}^{(1)}(N, x)$ in Table 2 is $x_{6,16}^{(1)}(N)$, which converges to $\xi_{1}=0.5$ and the corresponding quadrature weight tends to $\lambda_{1}=5$.

Example 2. Now we consider the signal

$$
x(m)=\sum_{j=1}^{3}\left(\gamma_{j} e^{i m \omega_{j}}+\gamma_{n_{0}+1-j} e^{i m \omega_{n_{0}+1-j}}\right),
$$

where $n_{0}=6, \omega_{1}=2 \pi-\omega_{6}=\pi / 3, \omega_{2}=2 \pi-\omega_{5}=\pi / 2, \omega_{3}=2 \pi-\omega_{4}=4 \pi / 5$, $\gamma_{1}=\gamma_{6}=10, \gamma_{2}=\gamma_{5}=4$ and $\gamma_{3}=\gamma_{4}=1$.

We expect that the positive interesting zeros of $P_{n}^{(1)}(N, x)$ and the corresponding quadrature weights, as $N$ tends to infinity, converge to the values

$$
\begin{array}{ll}
\xi_{1}=\cos (\pi / 6)=0.86602540 \cdots, & \lambda_{1}=100 \\
\xi_{2}=\cos (\pi / 4)=0.70710678 \cdots, & \lambda_{2}=16 \\
\xi_{3}=\cos (2 \pi / 5)=0.30901699 \cdots, & \lambda_{3}=1 .
\end{array}
$$

For this example, we analyze the zeros of $P_{14}^{(1)}(N, x)$ and $P_{15}^{(1)}(N, x)$ for increasing values of $N$. In Table 3, we give the positive zeros of $P_{14}^{(1)}(N, x)$ and the corresponding quadrature weights. By observing the quadrature weights that approach 0 , we can easily eliminate the uninteresting zeros $x_{1,14}^{(1)}(N), x_{2,14}^{(1)}(N), x_{5,14}^{(1)}(N)$ and $x_{7,14}^{(1)}(N)$. The remaining interesting zeros $x_{3,14}^{(1)}(N), x_{4,14}^{(1)}(N)$ and $x_{6,14}^{(1)}(N)$ seem to converge to the required limits $\xi_{1}, \xi_{2}$ and $\xi_{3}$, respectively. From the limits of the corresponding quadrature weights one can also recover the values of $\lambda_{1}, \lambda_{2}$ and $\lambda_{3}$.

TABle 3. Positive zeros of $P_{n}^{(1)}(N, x)$, for $n=14$, and the corresponding quadrature weights for the signal given by (15)

\begin{tabular}{|c|c|c|c|c|c|}
\hline$N$ & 4096 & 16384 & 65536 & \multicolumn{1}{c|}{262144} & 1048576 \\
\hline$x_{1, n}^{(1)}(N)$ & .989212594 & .989299970 & .989210625 & .989299581 & .989210502 \\
$x_{2, n}^{(1)}(N)$ & .906162462 & .906761986 & .906133429 & .906755808 & .906131609 \\
$x_{3, n}^{(1)}(N)$ & .865979840 & .866014421 & .866022554 & .866024717 & .866025225 \\
$x_{4, n}^{(1)}(N)$ & .707215505 & .707135396 & .707113588 & .707108570 & .707107206 \\
$x_{5, n}^{(1)}(N)$ & .607312636 & .611289737 & .607296488 & .611295467 & .607295454 \\
$x_{6, n}^{(1)}(N)$ & .309555993 & .309108278 & .309050983 & .309022710 & .309019119 \\
$x_{7, n}^{(1)}(N)$ & .150003490 & .142155835 & .150181242 & .142175633 & .150192479 \\
$\chi_{1, n}^{(1)}(N)$ & $.112888 e-1$ & $.287092 e-2$ & $.705654 e-3$ & $.179437 e-3$ & $.441037 e-4$ \\
$\chi_{2, n}^{(1)}(N)$ & $.153277 e+0$ & $.373171 e-1$ & $.960046 e-2$ & $.233339 e-2$ & $.600109 e-3$ \\
$\chi_{3, n}^{(1)}(N)$ & $.998523 e+2$ & $.999642 e+2$ & $.999907 e+2$ & $.999977 e+2$ & $.999994 e+2$ \\
$\chi_{4, n}^{(1)}(N)$ & $.160273 e+2$ & $.160061 e+2$ & $.160017 e+2$ & $.160003 e+2$ & $.160001 e+2$ \\
$\chi_{5, n}^{(1)}(N)$ & $.301059 e-1$ & $.774932 e-2$ & $.188245 e-2$ & $.484465 e-3$ & $.117656 e-3$ \\
$\chi_{6, n}^{(1)}(N)$ & $.100595 e+1$ & $.100141 e+1$ & $.100037 e+1$ & $.100008 e+1$ & $.100002 e+1$ \\
$\chi_{7, n}^{(1)}(N)$ & $.730947 e-2$ & $.198729 e-2$ & $.458248 e-3$ & $.124244 e-3$ & $.286460 e-4$ \\
\hline
\end{tabular}


TABLE 4. The nonnegative zeros of $P_{n}^{(1)}(N, x)$, for $n=15$ and the corresponding quadrature weights for the signal given by (15)

\begin{tabular}{|c|c|c|c|c|c|}
\hline$N$ & \multicolumn{1}{|c|}{65536} & \multicolumn{1}{c|}{131072} & \multicolumn{1}{c|}{262144} & \multicolumn{1}{c|}{524288} & \multicolumn{1}{c|}{1048576} \\
\hline$x_{1, n}^{(1)}(N)$ & .990694550 & .991069694 & .990707384 & .991109746 & .990694385 \\
$x_{2, n}^{(1)}(N)$ & .918162836 & .920315195 & .918158191 & .920506677 & .918161176 \\
$x_{3, n}^{(1)}(N)$ & .866024393 & .866025016 & .866025149 & .866025308 & .866025340 \\
$x_{4, n}^{(1)}(N)$ & .707388335 & .710944712 & .707148927 & .708477691 & .707125604 \\
$x_{5, n}^{(1)}(N)$ & .703238297 & .706967424 & .700700794 & .707010273 & .703489755 \\
$x_{6, n}^{(1)}(N)$ & .405875346 & .407626485 & .386511972 & .385318866 & .405829382 \\
$x_{7, n}^{(1)}(N)$ & .308955310 & .308988523 & .308993668 & .309005739 & .309013135 \\
$x_{8, n}^{(1)}(N)$ & .000000000 & .000000000 & .000000000 & .000000000 & .000000000 \\
$\chi_{1, n}^{(1)}(N)$ & $.635379 e-3$ & $.356439 e-3$ & $.162438 e-3$ & $.923051 e-4$ & $.397117 e-4$ \\
$\chi_{2, n}^{(1)}(N)$ & $.510492 e-2$ & $.243761 e-2$ & $.128986 e-2$ & $.614839 e-3$ & $.319084 e-3$ \\
$\chi_{3, n}^{(1)}(N)$ & $.999944 e+2$ & $.999961 e+2$ & $.999986 e+2$ & $.999990 e+2$ & $.999996 e+2$ \\
$\chi_{4, n}^{(1)}(N)$ & $.149311 e+2$ & $.569533 e+0$ & $.158983 e+2$ & $.105795 e+1$ & $.159183 e+2$ \\
$\chi_{5, n}^{(1)}(N)$ & $.107307 e+1$ & $.154316 e+2$ & $.102705 e+0$ & $.149423 e+2$ & $.819297 e-1$ \\
$\chi_{6, n}^{(1)}(N)$ & $.107399 e-2$ & $.481206 e-3$ & $.371597 e-3$ & $.173370 e-3$ & $.671996 e-4$ \\
$\chi_{7, n}^{(1)}(N)$ & $.999815 e+0$ & $.999950 e+0$ & $.999839 e+0$ & $.999932 e+0$ & $.999988 e+0$ \\
$\chi_{8, n}^{(1)}(N)$ & $.320445 e-3$ & $.146707 e-3$ & $.964100 e-4$ & $.450728 e-4$ & $.200286 e-4$ \\
& $.160042 e+2$ & $.160011 e+2$ & $.160010 e+2$ & $.160002 e+2$ & $.160002 e+2$ \\
\hline
\end{tabular}

In Table 4, we present the nonnegative zeros of $P_{15}^{(1)}(N, x)$. Again we can easily eliminate the uninteresting zeros $x_{1,15}^{(1)}(N), x_{2,15}^{(1)}(N), x_{6,15}^{(1)}(N)$ and $x_{8,15}^{(1)}(N)$, by observing the corresponding quadrature weights. The behavior of the quadrature weights $\chi_{3,15}^{(1)}(N)$ and $\chi_{7,15}^{(1)}(N)$ also indicate that $x_{3,15}^{(1)}(N)$ approaches the limit $\xi_{1}$ and $x_{7,15}^{(1)}(N)$ approaches the limit $\xi_{3}$.

It is rather difficult to say much about the individual behavior of the quadrature weights $\chi_{4,15}^{(1)}(N)$ and $\chi_{5,15}^{(1)}(N)$. However, as expected from Theorems 4.2 and 4.3 the sum $\chi_{4,15}^{(1)}(N)+\chi_{5,15}^{(1)}(N)$ tends to the limit $\lambda_{2}$. The numbers in the last row of Table 4 represent these sums.

\section{REFERENCES}

[1] G.S. Ammar, D. Calvetti and L. Reichel, Continuation methods for the computation of zeros of Szegö polynomials, Linear Algebra and its Appl., 249 (1996), 125-155. MR.97j:65081

[2] A.C. Berti and A. Sri Ranga, Companion orthogonal polynomials: some applications, Appl. Numer. Maths., 39 (2001), 127-149. MF,2002k:33006

[3] C.F. Bracciali, A.P. da Silva and A. Sri Ranga, Szegő polynomials: some relations to $L$ orthogonal and orthogonal polynomials, J. Comput. Appl. Math., 153 (2003), 79-88.

[4] R. Bressan, S.F. Menegasso and A. Sri Ranga, Szegö polynomials: quadrature rules on the unit circle and on [-1,1], Rocky Mountain J. Math., 33 (2003), 567-584.

[5] L. Daruis, O. Njåstad and W. Van Assche, Para-orthogonal polynomials in frequency analysis, Rocky Mountain J. Math., 33 (2003), 629-645.

[6] P. Delsarte and Y. Genin, The split Levinson algorithm, IEEE Trans. Acoust. Speech Signal Process, 34 (1986), 470-478. MR 87f:94007

[7] P. Delsarte and Y. Genin, An introduction to the class of split Levinson algorithms, in Numerical Linear Algebra, Digital Signal Processing and Parallel Algorithms (G.H. Golub 
and P. Van Dooren, eds.), NATO ASI Series F, Vol. 70, pp. 111-130, Springer-Verlag, 1991. MR $92 \mathrm{k}: 65076$

[8] W. Gautschi, Orthogonal polynomials - Constructive theory and applications, J. Comput. Appl. Math., 12/13 (1985), 61-76. MR87a:65045

[9] W.B. Jones, O. Njåstad and E.B. Saff, Szegő polynomials associated with Wiener-Levinson filters, J. Comput. Appl. Math., 32 (1990), 387-407. MR92e:94001

[10] W.B. Jones, O. Njåstad and W.J. Thron, Moment theory, orthogonal polynomials, quadrature, and continued fractions associated with the unit circle, Bull. London Math. Soc., 21 (1989), 113-152. MR90e:42027

[11] W.B. Jones, O. Njåstad, W.J. Thron and H. Waadeland, Szegő polynomials applied to frequency analysis, J. Comput. Appl. Math., 46 (1993), 217-228. MR94g:94003

[12] W.B. Jones, O. Njåstad and H. Waadeland, An alternative way of using Szegö polynomials in frequency analysis, in Continued Fractions and Orthogonal Functions (S.C. Cooper and W.J. Thron, eds.), Lecture Notes in Pure and Applied Mathematics, Vol. 154, pp. 141-152, Marcel Dekker, 1994. MR 95h:94001

[13] W.B. Jones and V. Peterson, Continued fractions and Szegö polynomials in frequency analysis and related topics, Acta Appl. Math., 61 (2000), 149-174. MF.2001f:65019

[14] W.B. Jones, W.J. Thron and H. Waadeland, A strong Stieltjes moment problem, Trans. Amer. Math. Soc., 261 (1980), 503-528. MF 81j:30055

[15] N. Levinson, The Wiener RMS (root mean square) error criterion in filter design and prediction, J. Math. Phys. Mass. Inst. Techn., 25 (1947), 261-278. MR8:391e

[16] K. Pan, A refined Wiener-Levinson method in frequency analysis, SIAM J. Math. Anal., 27 (1996), 1448-1453. MR97h:94002

[17] K. Pan and E.B. Saff, Asymptotics for zeros of Szegő polynomials associated with trigonometric polynomial signals, J. Approx. Theory, 71 (1992), 239-251. MR.94d:41013

[18] R.A. Sack and A.F. Donovan, An algorithm for Gaussian quadrature given modified moments, Numer. Math., 18 (1971/72), 465-478. MR46:2829

[19] G. Szegö, Orthogonal Polynomials, 4th ed., Amer. Math. Soc. Colloq. Publ., vol. 23, Amer. Math. Soc., Providence, RI, 1975. MR 51:8724

[20] W. Van Assche, Orthogonal polynomials in the complex plane and on the real line, in Fields Institute Communications 14: Special functions, $q$-series and related topics (M.E.H. Ismail et al., eds.), Amer. Math. Soc. (1997), 211-245. MR98i:33014

[21] N. Wiener, Extrapolation, Interpolation and Smoothing of Stationary Time Series, The Technology Press of the Massachusetts Institute of Technology and John Wiley and Sons, 1949. MR:11:118j

[22] A. Zhedanov, On some classes of polynomials orthogonal on arcs of the unit circle connected with symmetric orthogonal polynomials on an interval, J. Approx. Theory, 94 (1998), 73-106. MR2000a:42040

Departamento de Ciências de Computação e Estatística, ibilce, UnesPUniversidade Estadual Paulista, 15054-000 São José do Rio Preto, SÃo Paulo, Brazil

Department of Mathematics, University of Central Florida, Orlando, Florida 32816

Departamento de Ciências de Computação e Estatística, ibilce, UnesPUniversidade Estadual Paulista, 15054-000 São José do Rio Preto, SÃo Paulo, Brazil 